\title{
A two-step scheme for high-resolution regional atmospheric trace gas inversions based on independent models
}

\author{
C. Rödenbeck, C. Gerbig, K. Trusilova, and M. Heimann \\ Max Planck Institute for Biogeochemistry, Postfach 100164, 07701 Jena, Germany \\ Received: 1 September 2008 - Published in Atmos. Chem. Phys. Discuss.: 19 January 2009 \\ Revised: 23 June 2009 - Accepted: 23 June 2009 - Published: 29 July 2009
}

\begin{abstract}
Mixing ratio measurements of atmospheric tracers like $\mathrm{CO}_{2}$ can be used to estimate regional surface-air tracer fluxes using inverse methods, involving a numerical transport model. Currently available transport models are either global but rather coarse, or more accurate but only over a limited spatial and temporal domain. To obtain higher-resolution flux estimates within a region of interest, existing studies use zoomed or coupled models. The two-step scheme developed here uses global and regional models sequentially in separate inversion steps, coupled only via the data vector. This provides a nested atmospheric inversion scheme without the necessity of a direct coupled model implementation. For example, the scheme allows an easy nesting of Lagrangian models with their potential of very high resolution into global inversions based on Eulerian models.
\end{abstract}

\section{Introduction}

"Atmospheric transport inversions" are a tool to estimate the surface-atmosphere exchange of biogeochemical trace gases based on atmospheric mixing ratio measurements and a numerical transport model. This method is widely used, e.g., for atmospheric $\mathrm{CO}_{2}$ (Enting et al., 1995; Bousquet et al., 2000; Rödenbeck et al., 2003; Baker et al., 2006, and many others). Since atmospheric transport links any location on Earth within less than one year, the inversion problem is intrinsically global, such that a global transport model and a global flux representation need to be used. On the other hand, both atmospheric transport and the source/sink patterns of $\mathrm{CO}_{2}$ (or other biogeochemical species) have significant variability on fine spatial and temporal scales (less than kilometres or hours, especially over continents) - neglect-

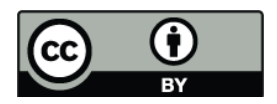

Correspondence to: C. Rödenbeck (christian.roedenbeck@bgc-jena.mpg.de) ing this variability leads to considerable errors in the flux retrievals (Gerbig et al., 2003a,b; van der Molen and Dolman, 2007; Pérez-Landa et al., 2007; Ahmadov et al., 2007). However, given present-day computing capabilities, atmospheric transport models are either global with grid resolutions of no finer than about $2^{\circ} \times 2^{\circ}$, or of finer scale (down to about $2 \mathrm{~km} \times 2 \mathrm{~km}$ ) but only for a limited spatial domain and temporal period. In the near future, we do not expect the availability of models that could combine high resolution and global/multi-year coverage.

Existing studies solve the problem by focusing on a domain of interest (DoI) and possibly a period of interest (PoI) over which fluxes and transport are finer resolved. This can be done using "zoomed" atmospheric transport models gradually refining the grid resolution towards the DoI (e.g., Peylin et al., 2005), or nested models with higher-resolution insets coupled along the boundary (e.g., Peters et al., 2007).

The two-step algorithm presented here has been designed with the following aims:

- The scheme is meant to allow completely independent models to be used for global and regional transport. "Independent" means that the two models do not need to be of similar type, and, even more importantly, the two models do not need to be run in a coupled mode (the implementation of nesting capabilities can be rather involved, and would be required for both the models and their adjoints). An envisaged application is the combination of a global Eulerian (gridded) model with a Lagrangian (backward trajectory) regional model to resolve the flux field in the vicinity of the observations with much higher spatial and temporal resolution at relatively low computational cost (Lin et al., 2003; Gerbig et al., 2003a).

- Nesting by any method poses several issues, that need to be solved to sufficient approximation. Within the DoI, for a prescribed surface flux field, different models (or

Published by Copernicus Publications on behalf of the European Geosciences Union. 
nests of the same model with different resolutions) will simulate different mixing ratio fields (e.g., Geels et al., 2007) - how to handle this mismatch? For a short PoI, how to spin up the atmospheric mixing ratio field from an unknown state? For example, Peylin et al. (2005) were able to solve the spin-up problem in a 1-month inversion using a SVD decomposition of the initial mixing ratio field. Additional degrees of freedom could also be introduced at the boundary (compare Lauvaux et al., 2008). The aim of the present scheme is to solve these issues as accurately as possible, but also with as few as possible changes to existing global or regional singlemodel inversion schemes.

This paper describes the two-step recipe, and demonstrates it with the TM3 transport model run on different spatial resolutions. The example region (DoI) is Europe where a relatively dense observation network is available.

\section{Method}

\subsection{Basic inversion scheme}

Before describing the two-step approach, we review the "ordinary" (global) inversion process, thereby also introducing the involved quantities. The method ("linear Bayesian inverse estimation") is used in many atmospheric trace gas studies. Some details of the present description refer to the implementation for $\mathrm{CO}_{2}$ by Rödenbeck et al. (2003) and its extensions described in Rödenbeck (2005), but the nesting scheme should also be applicable as an extension to other implementations.

The primary input to the flux estimation is the observed mixing ratios $\boldsymbol{c}_{\text {meas }}$ (vector of all individual measured values at different times and locations). Modelled mixing ratios $\boldsymbol{c}_{\text {mod }}$ that arise from a given temporally and spatially varying discretized flux field $\boldsymbol{f}$ are computed by an atmospheric transport model. Formally, they can be written as ${ }^{1}$

$\boldsymbol{c}_{\text {mod }}=\mathbf{A} \boldsymbol{f}+\boldsymbol{c}_{\text {ini }}$

in terms of a transport matrix $\mathbf{A}$. The values in $\boldsymbol{c}_{\text {mod }}$ are sampled in the model for every individual time and location where there is a measured value in $\boldsymbol{c}_{\text {meas }}$.

The inversion calculation seeks those fluxes $f$ that lead to the best match between observed and modelled mixing ratios, in the sense that the value of the cost function

$J_{c}=\frac{1}{2}\left(\boldsymbol{c}_{\text {meas }}-\boldsymbol{c}_{\text {mod }}\right)^{\mathrm{T}} \mathbf{Q}_{\boldsymbol{c}}^{-1}\left(\boldsymbol{c}_{\text {meas }}-\boldsymbol{c}_{\text {mod }}\right)$

\footnotetext{
${ }^{1}$ Here, the initial condition $\boldsymbol{c}_{\text {ini }}$ corresponds to a well-mixed atmosphere with a given initial tracer mixing ratio; the flux-related increments in mixing ratio since the start of the simulation will be denoted by

$\Delta \boldsymbol{c}_{\mathrm{mod}}=\boldsymbol{c}_{\mathrm{mod}}-\boldsymbol{c}_{\mathrm{ini}}$
}

is minimal. The (diagonal) matrix $\mathbf{Q}_{\boldsymbol{c}}$ introduces a weighting among the mixing ratio values (here involving assumed measurement uncertainty, location-dependent model uncertainty, and a data density weighting, Rödenbeck, 2005).

Generally, minimization of the above cost function would be an ill-posed problem, which is usually remedied by adding Bayesian a-priori constraints. This is done here by writing the flux field $f$ as

$\boldsymbol{f}=\boldsymbol{f}_{\text {fix }}+\mathbf{F} \boldsymbol{p}$

("linear statistical flux model"). The vector $\boldsymbol{p}$ represents the set of adjustable parameters, each of which acts as a multiplier to one of the columns of the matrix $\mathbf{F}$. These columns represent elementary spatio-temporal flux patterns (like elementary flux pulses or Fourier modes) composing the total flux. Mathematical stability of the flux estimation is ensured by adding a second term to the cost function,

$J=J_{c}+\frac{1}{2} \boldsymbol{p}^{\mathrm{T}} \boldsymbol{p}$

As, by construction, the parameters $\boldsymbol{p}$ a-priori have zero mean, unit variance, and are uncorrelated, this corresponds to introducing a Bayesian a-priori probability distribution for $f$ with mean ("best-guess") $f_{\text {fix }}$ and covariance matrix $\mathbf{Q}_{f, \text { pri }}=\mathbf{F F}^{\mathrm{T}}$.

The cost function $J$ is then minimized with respect to the parameters $\boldsymbol{p}$ (here using a Conjugate Gradient algorithm with re-orthogonalization, Rödenbeck, 2005).

\subsection{Nesting}

The two-step scheme to be presented considers nesting both in space and time:

Spatially, fluxes are meant to be estimated only within a domain of interest (DoI), but with finer resolution.

Temporally, the period of interest (PoI) might be as short as one year or less. The PoI is nested into the full period (FP) that includes at least some additional time for spin-up at the beginning, such that the artificial initial condition has been "forgotten" by the start of the PoI (here, the spin-up time is chosen to be one year, corresponding to the mixing time of the global atmosphere). (The temporal resolution of the flux is not increased as the iterative solution can technically handle any time step already).

Restricting the inversion calculation to the DoI/PoI makes the use of a high-resolution model (Lagrangian or mesoscale Eulerian) feasible, but then only the data information from inside the DoI/PoI is available. The nesting algorithm therefore needs to supply the missing information from the data outside the DoI or before the PoI, at least to a good approximation.

\subsection{Motivation of the two-step nesting scheme}

To motivate how the algorithm will do this, we first consider in which way our target quantity - the fluxes $f^{\text {reg }}(\boldsymbol{x}, t)$ within 
the DoI/PoI - is reflected in the regional mixing ratio signals. The (regional) mixing ratio field $c^{\text {reg }}$ within the DoI/PoI is written as a superposition

$c^{\mathrm{reg}}(\boldsymbol{x}, t)=c_{\text {trans }}^{\mathrm{reg}}(\boldsymbol{x}, t)+c_{\mathrm{cis}}^{\mathrm{reg}}(\boldsymbol{x}, t)$

of two contributions:

1. The "cis"-contribution $c_{\text {cis }}^{\text {reg }}(\boldsymbol{x}, t)$ is the mixing ratio of all tracer substance that originated from sources/sinks $f^{\text {reg }}\left(\boldsymbol{x}^{\prime}, t^{\prime}\right)$ located within the DoI and occurring during the PoI, and that arrived at location $\boldsymbol{x}$ and time $t$ without ever leaving the DoI (hereafter called I $\rightarrow$ I pathways);

2. The "trans"-contribution $c_{\text {trans }}^{\text {reg }}(\boldsymbol{x}, t)$ is the mixing ratio of all tracer substance that entered the DoI/PoI across its boundary, either originating from fluxes outside the $\mathrm{DoI} /$ before the $\mathrm{PoI}(\mathrm{O} \rightarrow \mathrm{I}$ pathways $)$, or originating from fluxes inside the DoI/PoI but temporary having left it ( $\mathrm{I} \rightarrow \mathrm{O} \rightarrow \mathrm{I}$ pathways).

This split of the regional mixing ratio field exists because the underlying continuity equation is linear (see Appendix A for details).

Importantly, the trans-contribution depends only very weakly on the regional fluxes $f^{\text {reg }}(\boldsymbol{x}, t)$ : Except for some immediate re-circulation close to the boundary, signals related to $f^{\text {reg }}(\boldsymbol{x}, t)$ only re-enter the domain as part of the global background, with any structure smoothed out. Therefore, the regional fluxes $f^{\text {reg }}(\boldsymbol{x}, t)$ essentially lead to a large-scale offset to $c_{\text {trans }}^{\text {reg }}(\boldsymbol{x}, t)$ only. In terms of local gradients or variability, it is only the cis-contribution $c_{\text {cis }}^{\text {reg }}(\boldsymbol{x}, t)$ that reflects the fine structure of $f^{\text {reg }}(\boldsymbol{x}, t)$.

An inversion as sketched in Sect. 2.1 above inverts the link between the global fluxes and the global mixing ratio field: the gradients and changes in a set of mixing ratio data are used to estimate the fluxes $f(\boldsymbol{x}, t)$. As the regional fluxes $f^{\text {reg }}(\boldsymbol{x}, t)$ are essentially linked to the cis-contribution only, the idea of the two-step scheme proposed here is to first (approximately) remove the influence of the smooth trans-contribution from the regional data. Then, in a second step, the regional inversion can simply be done with a zero-influx regional transport model which just simulates the cis-contribution $c_{\text {cis }}^{\text {reg }}(\boldsymbol{x}, t)$ (Appendix A).

In the remainder of this Sect. 2, the scheme is presented just algorithmically, while Sect. 4 will illustrate its functioning, discuss the involved errors, and quantify the goodness of approximation.

\subsection{The recipe of the two-step nesting scheme}

To facilitate the following description, see Fig. 1 for an overview. The scheme involves several independent transport models as defined in Table 1.

\subsubsection{Step 1}

The first step consists in an ordinary global inversion as of Sect. 2.1, done with all available data and the global transport model $\mathbf{A}_{\text {coarse }}^{\text {glob }}$ (see Table 1). Its result is the global coarseresolution flux estimates $\boldsymbol{f}_{1}$ over the FP.

\subsubsection{Intermediate step: The "remaining mixing ratio"}

Based on the results $f_{1}$ of the step-1 inversion, two forward runs are performed:

1. A normal run of the global transport model (over the FP), yielding modelled mixing ratio increments

$\Delta \boldsymbol{c}_{\text {mod }_{1}}=\mathbf{A}_{\text {coarse }}^{\text {glob }} \boldsymbol{f}_{1}$

(By construction, $\boldsymbol{c}_{\bmod _{1}}=\Delta \boldsymbol{c}_{\bmod _{1}}+\boldsymbol{c}_{\text {ini }}$ will closely match the data $\boldsymbol{c}_{\text {meas }}$.)

2. A run of the manipulated "regional" coarse-grid model $\mathbf{A}_{\text {coarse }}^{\text {reg }}$ (Table 1), starting with zero mixing ratio at the beginning of the PoI. The resulting modelled mixing ratio increments

$\Delta \boldsymbol{c}_{\text {mod }_{1}, \mathrm{cis}}=\mathbf{A}_{\text {coarse }}^{\text {reg }} \boldsymbol{f}_{1}$

will only give the cis-contributions $(\mathrm{I} \rightarrow \mathrm{I})$ inside the $\mathrm{DoI} / \mathrm{PoI}$ (and can be considered zero at all sites outside).

Then, a "remaining mixing ratio"

$\Delta \boldsymbol{c}_{\text {remain }}=\boldsymbol{c}_{\text {meas }}-(\underbrace{\Delta \boldsymbol{c}_{\text {mod }_{1}}-\Delta \boldsymbol{c}_{\text {mod }_{1}, \text { cis }}}_{\Delta \boldsymbol{c}_{\text {mod }_{1} \text {, trans }}}+\boldsymbol{c}_{\text {ini }})$

is calculated for all observational sites inside the DoI/PoI. It represents the data diminished by the trans-contribution to the mixing ratio as calculated by the global model from the fluxes $\boldsymbol{f}_{1}$.

\subsubsection{Step 2}

Finally, a second inversion run is performed, that differs from the step-1 inversion in two main aspects:

- The coarse model $\mathbf{A}_{\text {coarse }}^{\text {glob }}$ is replaced by the highresolution regional transport model $\mathbf{A}_{\text {fine }}^{\text {reg }}$, and all fluxes are represented on fine resolution.

- The data vector $\boldsymbol{c}_{\text {meas }}$ is replaced by the "remaining mixing ratios" $\Delta c_{\text {remain }}$ from the DoI/PoI.

In addition, some secondary changes are required, because the restriction of the fluxes to the DoI and PoI affects the a-priori correlation structure of the inversions. Details are described in Appendix B. In all other aspects, step 2 is technically just an ordinary inversion as step 1 .

The results $\boldsymbol{f}_{2}$ of the step- 2 inversion give the final highresolution flux estimates within the DoI/PoI. 


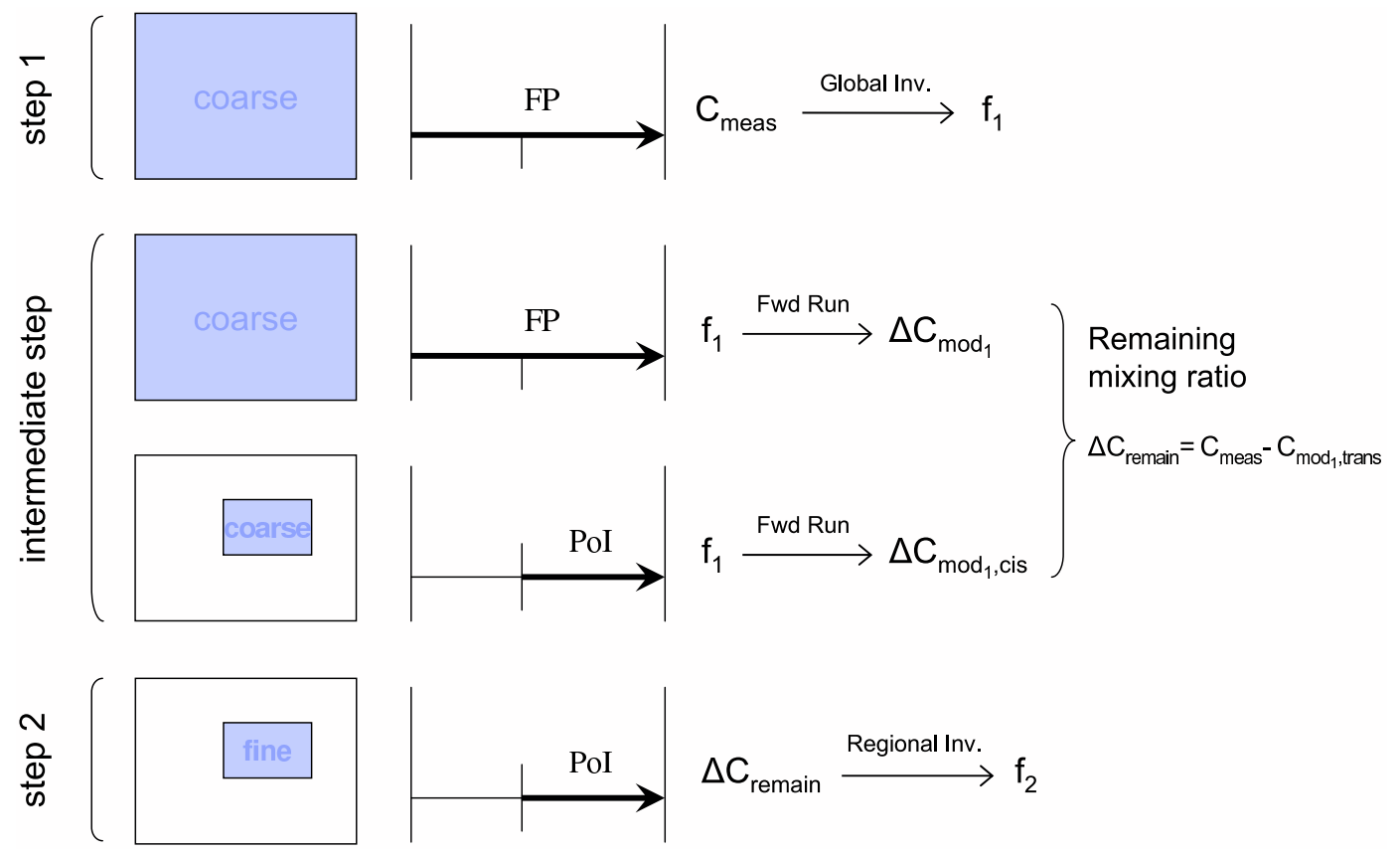

Fig. 1. Schematic overview of the individual steps of the algorithm described in Sect. 2.4.

Table 1. Definition of the independent transport models that play a role in the 2-step nesting scheme (symbolized by their transport matrices).

\begin{tabular}{|c|c|}
\hline $\mathbf{A}_{\text {coarse }}^{\text {glob }}$ & $\begin{array}{l}\text { A global transport model on a coarser resolution, that } \\
\text { can be run for the full multi-year period (FP). }\end{array}$ \\
\hline $\mathbf{A}_{\text {fine }}^{\mathrm{reg}}$ & $\begin{array}{l}\text { A higher-resolution model simulating only the DoI } \\
\text { (and only running during the PoI). The model is run } \\
\text { with simple zero boundary (and initial) conditions, } \\
\text { such that all tracer substance leaving the DoI is lost, } \\
\text { and no tracer (re-)enters over the boundary (i.e., it only } \\
\text { simulates the cis-part of the mixing ratio field arising } \\
\text { from I } \rightarrow \text { I pathways). }\end{array}$ \\
\hline $\mathbf{A}_{\text {coarse }}^{\text {reg }}$ & $\begin{array}{l}\text { A manipulated version of the global model } \mathbf{A}_{\text {coarse }}^{\text {glob }} \text {, } \\
\text { which mimics the regional model in only simulating } \\
\text { the cis-part of the mixing ratio field arising from } \mathrm{I} \rightarrow \mathbf{I} \\
\text { pathways (implemented here by setting the } 3 \mathrm{D} \text { mix- } \\
\text { ing ratio field outside the DoI/PoI to zero after every } \\
\text { transport or emission time step, see Appendix A). }\end{array}$ \\
\hline $\mathbf{A}_{\text {fine }}^{\text {glob }}$ & $\begin{array}{l}\text { A hypothetical transport model that has both higher } \\
\text { resolution and global coverage, as the conceptual } \\
\text { benchmark. }\end{array}$ \\
\hline
\end{tabular}

\subsection{Demonstration of the scheme}

The feasibility and the errors of the scheme have been checked by numerical test cases, using a $\mathrm{CO}_{2}$ inversion as an example.
The conceptual benchmark for any nesting scheme are the results of an (ordinary) inversion with the global highresolution transport model $\mathbf{A}_{\text {fine }}^{\text {glob }}$ (Table 1). Of course, this will not be feasible computationally in a real application. The global high-resolution flux estimates from the benchmark will be denoted $f_{\mathrm{B}}$, and the mixing ratios modelled from these fluxes using the same global high-resolution model are $\Delta \boldsymbol{c}_{\text {mod }_{\mathrm{B}}}=\mathbf{A}_{\text {fine }}^{\text {glob }} \boldsymbol{f}_{\mathrm{B}}$ with $\Delta \boldsymbol{c}_{\text {mod }_{\mathrm{B}} \text {, cis }}=\mathbf{A}_{\text {fine }}^{\text {reg }} \boldsymbol{f}_{\mathrm{B}}$ and $\Delta \boldsymbol{c}_{\text {mod }_{\mathrm{B}} \text {, trans }}=\Delta \boldsymbol{c}_{\text {mod }_{\mathrm{B}}}-\Delta \boldsymbol{c}_{\text {mod }_{\mathrm{B}} \text {, cis }}$ being their cis- and transcontributions, respectively.

In the example cases, the transport model TM3 (Heimann and Körner, 2003) driven by NCEP reanalysis (Kalnay et al., 1996) has been used. As this model can be run on different spatial resolutions, $\mathbf{A}_{\text {coarse }}^{\text {glob }}$ is provided by the TM3 model on a coarser resolution, while TM3 on a finer resolution does provide a benchmark model $\mathbf{A}_{\text {fine }}^{\text {glob }}$ here. A "regional model" $\mathbf{A}_{\text {fine }}^{\text {reg }}$ is obtained by applying the cut-out manipulation (as for $\mathbf{A}_{\text {coarse }}^{\text {reg }}$, see Table 1 and Appendix A) to the higher-resolution model $\mathbf{A}_{\text {fine }}^{\text {glob }}$. The particular TM3 resolutions used in the examples are given in Table 2, together with the different time periods of the runs.

Two tests (A and $\mathrm{B}$ ) are presented in this paper, that mainly differ in the kind of data: Test B uses real data, measured by various institutions. There are both flask data (mainly weekly sampling) and in-situ data (hourly averages, selected for day time and variability less than $1 \mathrm{ppm} / \mathrm{h}$ ). In contrast, test $\mathrm{A}$ uses synthetic data created by a forward run of the global higher-resolution benchmark model $\mathbf{A}_{\text {fine }}^{\text {glob }}$, such that the correct answer of the inversion is explicitly known. The 
Table 2. Characteristics of the two test inversions (for their detailed meaning see Sect. 2)

\begin{tabular}{lcc}
\hline & Test A & Test B \\
\hline TM3 res. ${ }^{\text {a }} \mathbf{A}_{\text {coarse }}^{\text {glob }} \mathbf{A}_{\text {coarse }}^{\text {reg }}$ & $\approx 8^{\circ} \times 10^{\circ} \times 9$ & $\approx 8^{\circ} \times 10^{\circ} \times 9$ \\
TM3 res. $\mathbf{A}_{\text {fine }}^{\text {reg }}, \mathbf{A}_{\text {fine }}^{\text {glob }}$ & $\approx 4^{\circ} \times 5^{\circ} \times 19$ & $\approx 1.8^{\circ} \times 1.8^{\circ} \times 28$ \\
Period of interest (PoI) & $2000-2006$ & 2006 \\
Full period (FP) $^{\text {b }}$ & $\begin{array}{c}1999-2006 \\
\text { synthetic }\end{array}$ & 2005-2006 \\
measurements \\
\hline
\end{tabular}

${ }^{\text {a }}$ Resolution given as latitude $\times$ longitude $\times$ number of layers

b Years inclusive

${ }^{\mathrm{c}}$ Synthetic mixing ratios are used at the same time instants and locations as the measurements

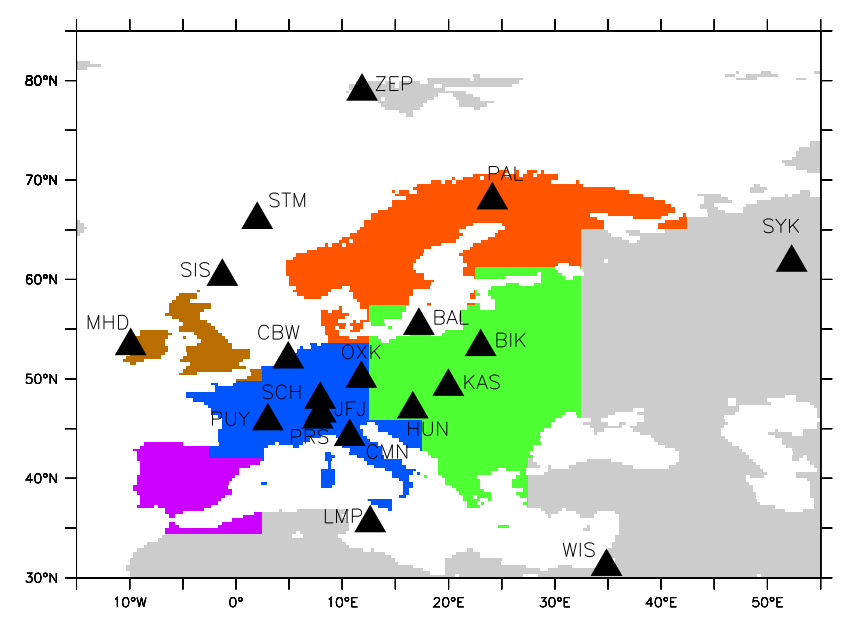

Fig. 2. Map of the domain of interest (DoI) of the example inversions, showing the measurement sites used in the estimation, as well as the integration regions for post-processing.

fluxes used to create the pseudo-data ("known truth") comprise daily fossil fuel emissions, terrestrial net ecosystem exchange (NEE), and ocean exchange ${ }^{2}$. Importantly, the time points and locations of the synthetic data are the same as those of the real data, such that they represent the same amount of atmospheric information as in a real inversion.

The domain of interest (DoI) in both tests is Europe and some surrounding areas $\left(15^{\circ} \mathrm{W}-55^{\circ} \mathrm{E}, 30^{\circ} \mathrm{N}-85^{\circ} \mathrm{N}\right)$. The measurement sites are shown in Fig. 2 (Europe only; there

2 Daily terrestrial NEE is taken from the BiomeBGC model (Trusilova and Churkina, 2008); daily fossil fuel emissions are from Olivier and Berdowski (2001) (with weekly and seasonal distribution functions), temporally extrapolated according to BP statistics (BP); and oceanic $\mathrm{CO}_{2}$ fluxes come from an inversion of ocean interior carbon data (sum of the anthropogenic fluxes from Mikaloff Fletcher et al. (2006), the preindustrial fluxes from Mikaloff Fletcher et al. (2007), and the river fluxes of Jacobson et al. (2007)). The exact choice of the "known truth" is however not critical. are 68 sites globally). ${ }^{3}$ As target quantity of the inversions, we take the temporal variations of the fluxes integrated over 5 parts of the DoI (the colored regions in Fig. 2). Temporally, we will consider 3-monthly filtered anomalies or unfiltered fluxes, respectively.

The inversion set-up (used for both tests) is very similar to that described in Rödenbeck (2005). Land fluxes are estimated on pixel resolution, with exponentially decaying a-priori correlations on a scale of around $1000 \mathrm{~km}$ (ocean: $2 \times 2$ aggregated pixels and around $1300 \mathrm{~km}$ correlation scale). Temporally, the fluxes have daily resolution, with a-priori correlations on a weekly scale. Fixed (a-priori) fluxes comprise annual fossil fuel emissions (Olivier and Berdowski, 2001), a constant NEE field (long-term mean of the LPJ model, Sitch et al., 2000), and a constant ocean exchange field (from Gloor et al., 2003). The main difference to Rödenbeck (2005) is a slightly tighter a-priori constraint (sigmas smaller by factor 2.83). Moreover, there are no extra seasonal components, as they would make less sense for a 1 -year inversion as in test B.

\section{Results}

\subsection{Test $\mathbf{A}$ - inverting synthetic data}

Figure 3 shows flux time series from within the chosen DoI/PoI to be compared for test A. The results of a global inversion using the global higher-resolution model $\mathbf{A}_{\text {fine }}^{\text {glob }}$ (orange line) are taken as the benchmark fluxes to be approximated as well as possible. A global inversion with the coarser model $\mathbf{A}_{\text {coarse (thin blue line) differs considerably from this }}^{\text {glo }}$ benchmark, a difference entirely due to the transport model errors. However, when going from this step- 1 inversion to the step-2 results $\boldsymbol{f}_{2}$ of the nesting scheme (thick purple line), the error is considerably reduced. Although the agreement in the temporal features with the benchmark is not perfect, the differences are now rather small compared to the temporal flux variability itself (see in particular the temporal standard deviations in the right panel).

In addition, the errors arising from nesting can be set into perspective with respect to the general errors of the inversion: Since synthetic data have been inverted here, the correct answer should be a reproduction of the fluxes ("known truth", black line) that have been used in the forward run creating the pseudo-data. Even the benchmark inversion - for which forward run and inversion use the same transport model such that transport is effectively perfect - is not fully able to reproduce the "known truth", reflecting inherent limitations of the inversion: the available information in the atmospheric

\footnotetext{
${ }^{3}$ Note that the data records of some sites do not span the entire time period of test A. In a real inversion, this may lead to artificial variations in time (Rödenbeck et al., 2003), but it should not be a problem here as it would affect benchmark and two-step results in approximately the same way.
} 
data set is incomplete (the mixing ratio is only known at discrete locations and times), and the adjustable degrees of freedom built into the estimation do not contain all features of the "known truth" (such as the variability on scales smaller/faster than the applied a-priori spatial/temporal correlations). Important in the present context, however, is that the difference between the step- 2 results and the benchmark are similar or smaller than their deviation from the "known truth": Thus, the nesting does not add a significant error to these inherent limitations that even the global high-resolution inversion faces.

\subsection{Test $\mathbf{B}$ - inverting real data}

This example tests the scheme in a situation closer to the real application: inverting measured data, using a higher resolution for the TM3 model in step 2, and running the highresolution step only over 1 year. The results are shown without temporal filtering in Fig. 4. Again, this test shows the clear improvements of the nested results over the results of the coarser-grid inversion. In terms of the temporal standard deviations, the deviations of the results of the step- 2 inversion from the benchmark are more than an order of magnitude smaller than the signal.

\section{Discussion}

\subsection{How does the scheme work?}

In order to illustrate the functioning of the scheme and the nature of the approximations involved, Fig. 5 (right panels) shows mixing ratio time series at the example site Schauinsland located well in the interior of the DoI. By construction of the inversion (step 1), the full modelled mixing ratios $\boldsymbol{c}_{\text {mod }_{1}}$ (upper panel, green) calculated from the optimized fluxes $\boldsymbol{f}_{1}$ fit the data $\boldsymbol{c}_{\text {meas }}$ (red) well, including the highfrequency variability. In constrast, the trans-contribution $\boldsymbol{c}_{\text {mod }_{1} \text {, trans }}$ to the mixing ratio (blue) is much smoother: It only represents signals advected from the boundary which have substantially been homogenized by atmospheric mixing already. The trans-contribution does contain some synopticscale variability related to transport, but all mixing ratio variability related to the detailed structure and variability of the fluxes in the near field is contained in the cis-contribution (see lower panel). Therefore, the cis-part of the mixing ratio contains essentially the full information about the regional fluxes. This reflects that local gradients (and shortterm changes) in the mixing ratio field mainly arise from local sources or sinks, while tracer substance advected from far away mainly leads to an offset only. Therefore, an inversion based only on the cis-part of the mixing ratio is still informed enough to retrieve the local flux structure.

What is subtracted from the data is only those contributions that cannot be handled by the zero-influx regional model. These contributions include, obviously, any responses to fluxes from outside the DoI or from before the PoI [O $\rightarrow$ I pathways]. Somewhat more subtle, however, they also include responses to fluxes from inside the DoI/PoI that temporarily left the DoI $[\mathrm{I} \rightarrow \mathrm{O} \rightarrow \mathrm{I}$ pathways], re-entering the DoI mainly as part of the background mixing ratio, after having travelled around the globe. The reason why it is not enough to eliminate the $\mathrm{O} \rightarrow \mathrm{I}$ contributions, becomes most obvious considering the long-term mixing ratio trend: The global trend is determined by the fluxes from both outside and inside the DoI. This trend needs to be subtracted completely because the regional model cannot accumulate any tracer over the years.

However, how well does the "remaining mixing ratio" $\Delta \boldsymbol{c}_{\text {remain }}$ used in step 2 approximate the cis-part, given that the subtracted trans-part is calculated from the coarse-model step 1? For the example site, Fig. 6 compares $\Delta \boldsymbol{c}_{\text {remain }}$ to what it should be according to the global high-resolution model (i.e., its counterpart in the benchmark inversion). The deviation is indeed much smaller than the signal. This can be understood as a general feature because (1) the full modelled mixing ratio $\boldsymbol{c}_{\text {mod }_{1}}$ already has a relatively good match to the data $\boldsymbol{c}_{\text {meas }}$ by construction of the optimization, even if there are considerable errors in the fluxes $f_{1}$ (see Fig. 5, upper panel, green vs. red) ${ }^{4}$, and (2) the most error-prone contributions to $c_{\text {mod }_{1}}$ are the near-field contributions, which are not part of $\boldsymbol{c}_{\text {mod }_{1} \text {, trans }}$ and therefore are cancelling out in $\Delta \boldsymbol{c}_{\text {remain }}$, at least away from the boundary. $\Delta \boldsymbol{c}_{\text {remain }}$ is thus a suitable approximation to the cis-contribution of the mixing ratio. Tests $\mathrm{A}$ and $\mathrm{B}$ confirm this: If $\Delta \boldsymbol{c}_{\text {remain }}$ was substantially contaminated by errors from the coarse model, then the step- 2 results in tests A and B would have deviated from the benchmark results in a similar order of magnitude as the step-1 results do. This is clearly not the case.

In addition to errors in $\Delta \boldsymbol{c}_{\text {remain }}{ }^{5}$, the deviations of the step2 results from the benchmark also arise from other issues:

- The a-priori covariance of the fluxes close to the boundary is altered due to edge effects (disruption of correlations, some changes in local a-priori uncertainly intervals). This problem is less severe with suitable choice of the DoI (see below).

\footnotetext{
${ }^{4}$ As an aside, this also confirms that the "remaining mixing ratio" has to be calculated by the same (coarse) model as used in the step-1 inversion: Using higher resolution in Eqs. (7) and (8) might seem preferrable, but would in fact cause a mismatch between inverse and forward runs, thereby increasing rather than decreasing the errors.

${ }^{5}$ The fraction of the deviations that are due to errors in $\Delta \boldsymbol{c}_{\text {remain }}$ has been quantified by a modified step 2 where $\Delta \boldsymbol{c}_{\text {remain }}$ has been calculated from the high-resolution model (benchmark) rather than the coarse one (step 1). In terms of the standard deviations as of Fig. 3 right panel, the deviations of this modified step 2 are smaller than those of the original step 2 by about $2 / 3$ in Western Europe (closer to the inflow boundary), and by about $1 / 5$ in the other regions.
} 

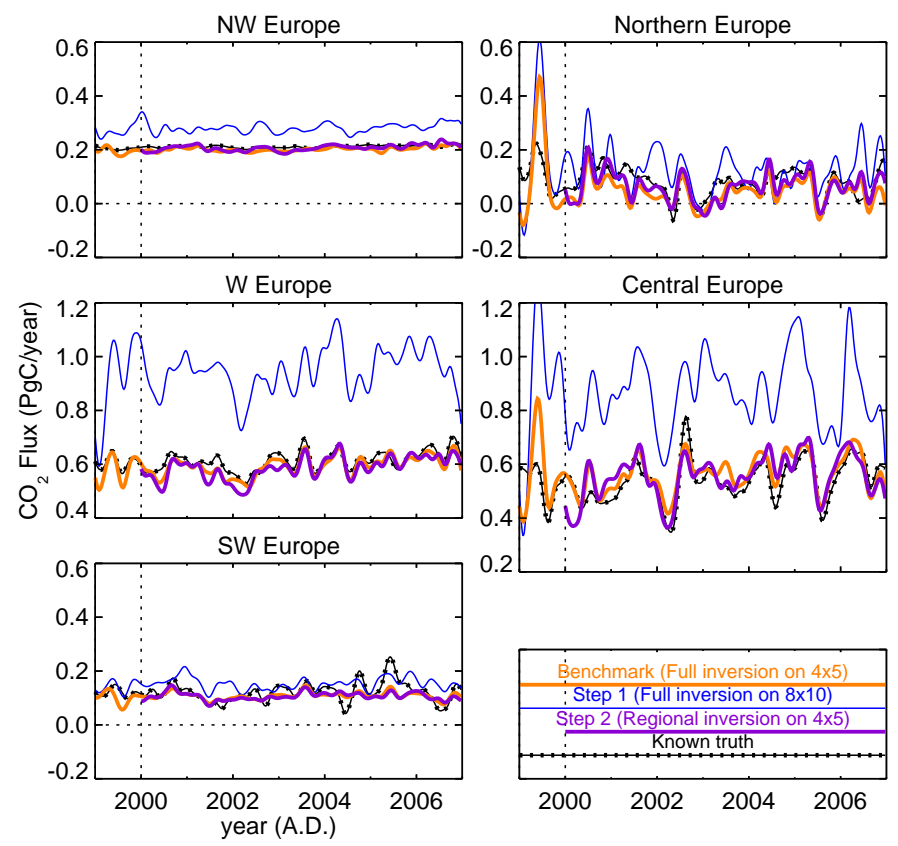

0.2
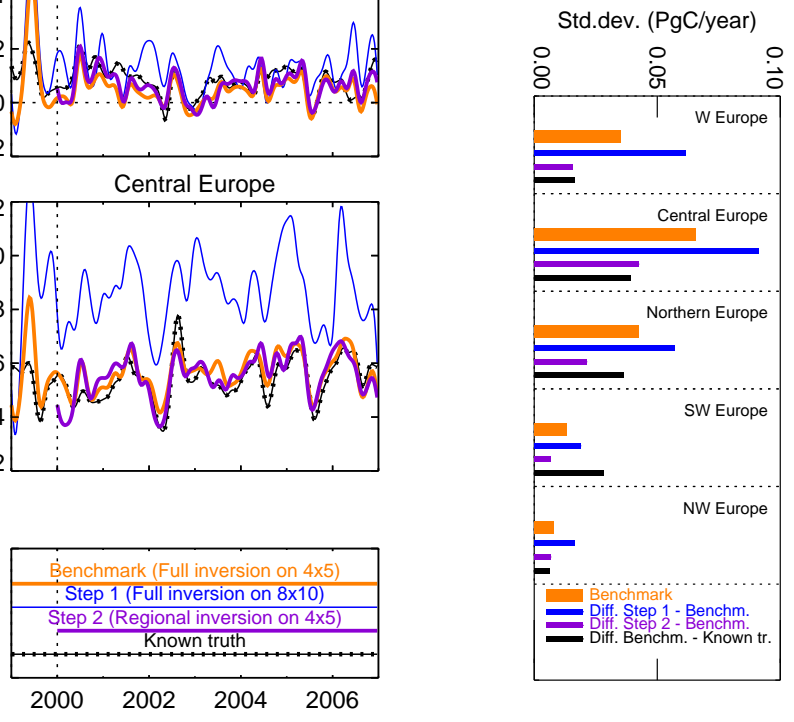

Fig. 3. Results of test A: Time series: Comparing the results of the benchmark inversion, steps 1 and 2 of the nested inversion, and the "known truth" used to create the synthetic data. Fluxes have been integrated over 5 parts of Europe (colored regions in Fig. 2), deseasonalized (by subtracting the mean seasonal cycle over 2001-2005 [incl.]), and 3-monthly filtered (Gaussian spectral weights).

Bars: Temporal standard deviations of the differences between the step-1 results, the step-2 results, or the "known truth", and the benchmark results (narrow bars). The standard deviation of the benchmark fluxes themselves is given as a reference of the signal size (wide bar). (Temporal standard deviations have been calculated from the filtered fluxes over the PoI; they do not incorporate the differences in mean.)
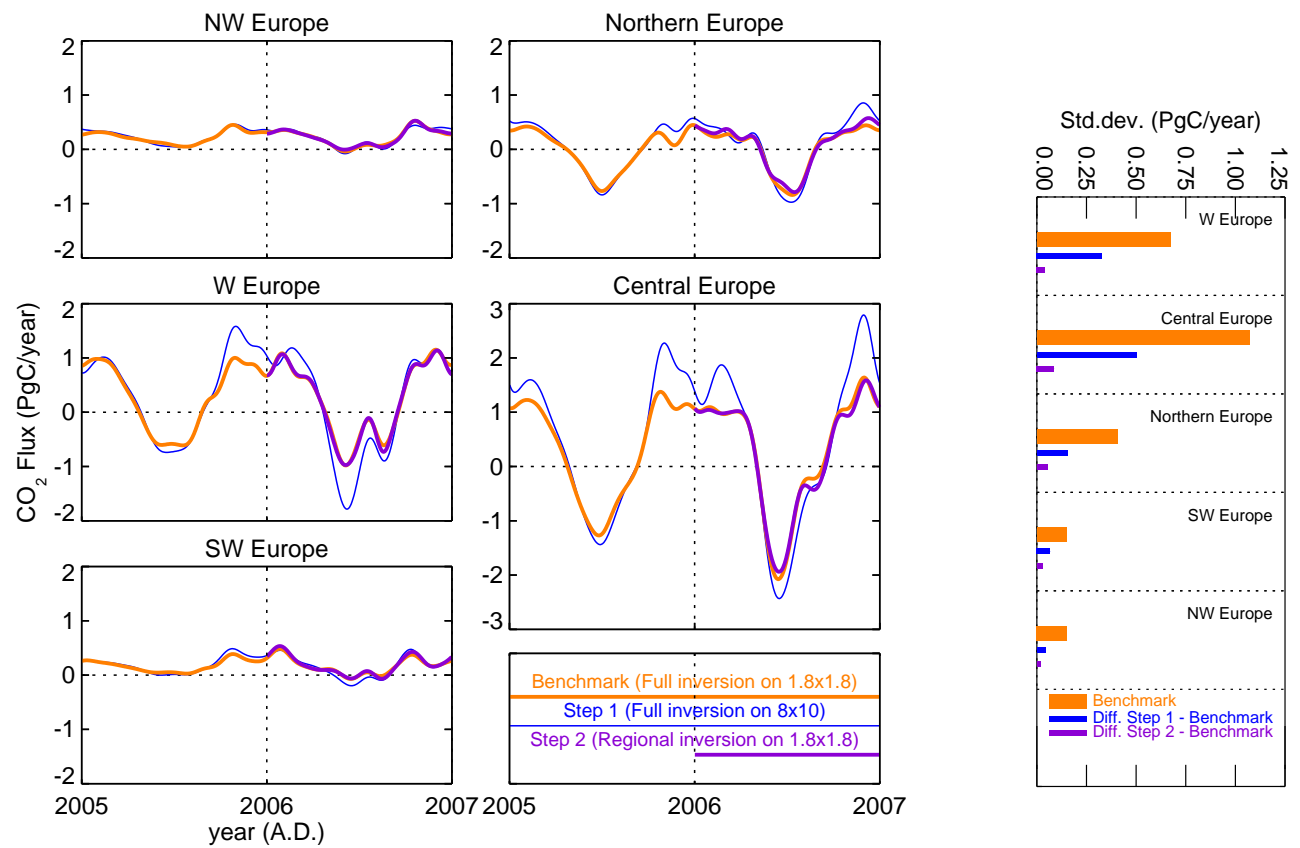

Fig. 4. Results of test B. Fluxes have been integrated over 5 parts of Europe as in Fig. 3, but not filtered temporally. 

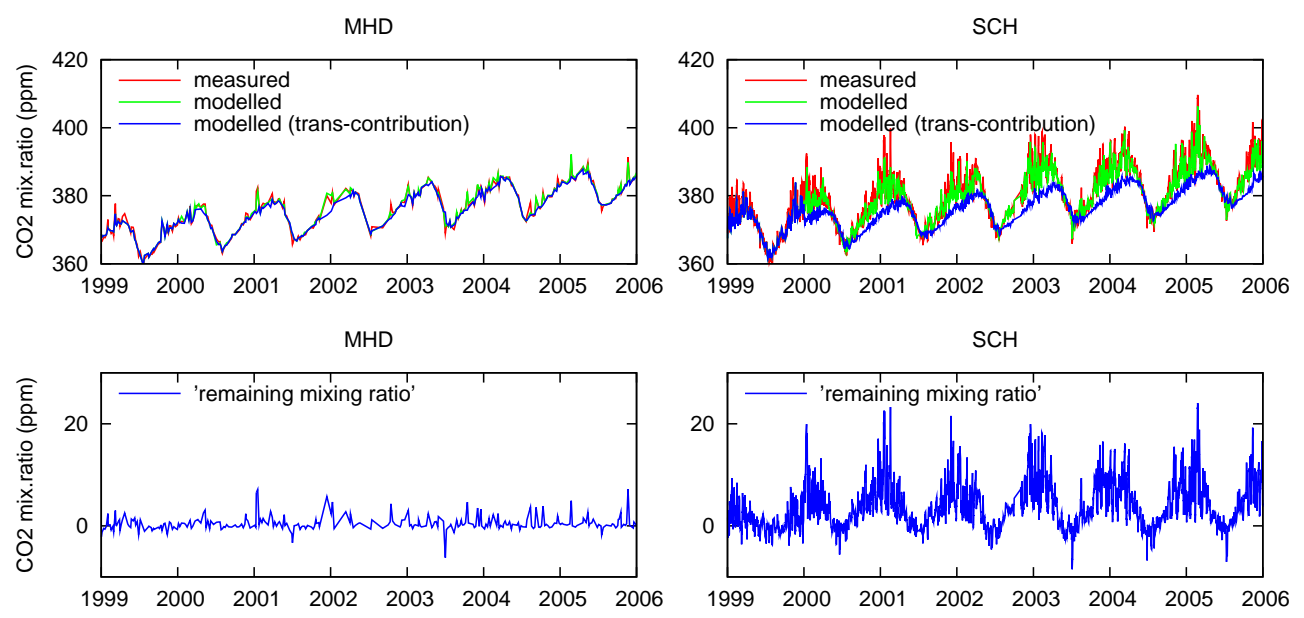

Fig. 5. Examples to illustrate the construction of the "remaining mixing ratio", at Mace Head close to the western inflow boundary (MHD, flask data) and at Schauinsland in the center of the DoI ( $\mathrm{SCH}$, hourly data). Top panels: Time series of measured mixing ratio ( $\boldsymbol{c}_{\mathrm{meas}}$, red),

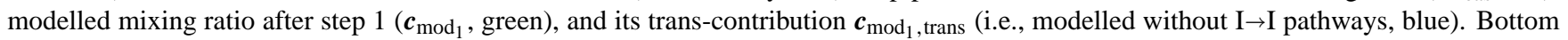
panels: The "remaining mixing ratio" ( $\left.\boldsymbol{c}_{\text {meas }}-\boldsymbol{c}_{\text {mod }_{1}, \text { trans }}\right)$.
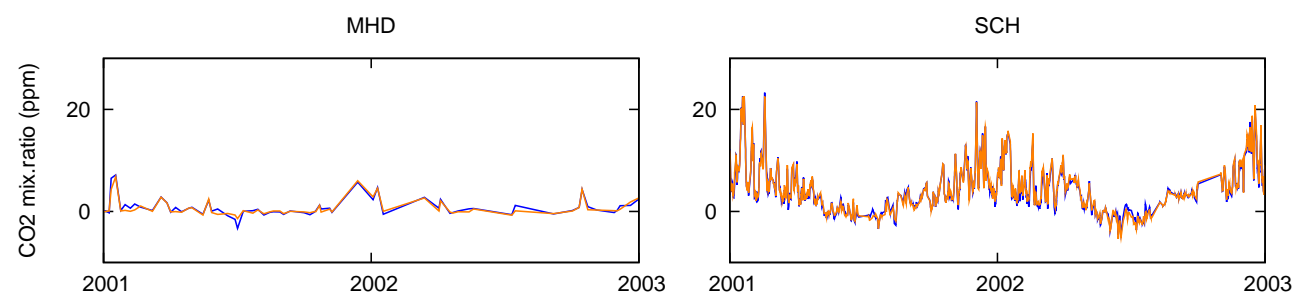

Fig. 6. Comparison of the "remaining mixing ratio" as calculated in step 1 ( $\boldsymbol{c}_{\text {meas }}-\boldsymbol{c}_{\text {mod }_{1} \text {, trans }}$, blue as in Fig. 5) with the corresponding quantity from the benchmark inversion $\left(\boldsymbol{c}_{\text {meas }}-\boldsymbol{c}_{\text {mod }_{\mathrm{B}}, \text { trans }}\right.$, orange).

- The inversion misses the degrees of freedom just outside of inflow boundaries for satisfying constraints at nearby sites. However, $\Delta \boldsymbol{c}_{\text {remain }}$ is correspondingly smaller at these sites (as at Mace Head shown in the left panels of Fig. 5), because the response to the fluxes outside is

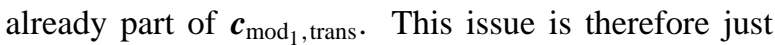
part of the errors of $\Delta \boldsymbol{c}_{\text {remain }}$ discussed above.

- The term $\boldsymbol{c}_{\text {mod }_{1} \text {, trans }}$ in $\Delta \boldsymbol{c}_{\text {remain }}$ (Eq. 8) statistically depends on the data and the prior fluxes also inside the DoI, causing two problems: (1) The (co)variances of $\Delta \boldsymbol{c}_{\text {remain }}$ differ from those of $\boldsymbol{c}_{\text {meas }}$, yet step 2 uses the same data uncertainty matrix $\mathbf{Q}_{c}$; and (2) data and prior fluxes in step 2 are not fully independent statistically, thereby violating assumptions of Bayesian inference. While the level of agreement between the inferred fluxes to the benchmark in tests A and B already indicates that the effect of these problems is sufficiently small, we examined the calculated uncertainties of the a-posteriori fluxes (Fig. 8) because those would be affected by wrong (co)variances more strongly than the a- posteriori fluxes themselves. Nonetheless, Fig. 8 reveals only very small deviations of the a-posteriori uncertainties as calculated in the step- 2 inversion relative to those calculated in the benchmark: The deviations are not larger than the small changes in a-priori (co)variances due to the spatial cut-out. (Larger deviations do occur for areas closer to the eastern boundary of the DoI, but this is where the inversion fails anyway due to lack of data.) This is again related to the fact that the flux features in the interior of the DoI are inferred by the inversion from local mixing ratio gradients/changes, the statistical properties of which are hardly altered by the subtraction of the trans-part that is strongly correlated across large scales. (Further confirmation comes from the fact that the a-posteriori correlations between the fluxes $f_{1}$ inside and outside of the DoI are small (not shown).)

- The coarse-grid regional model $\mathbf{A}_{\text {coarse }}^{\text {reg }}$ needed in Eq. (8) has been emulated by the global model with the DoI cut out (Table 1). This may create some mismatch error if 
the lines of the coarse grid do not coincide with the DoI boundaries (as is the case here because the finer TM3 grid is not a subdivision of the coarse one).

\subsection{Applicability of the scheme}

In tests $\mathrm{A}$ and $\mathrm{B}$, the scheme provided a good approximation to the benchmark fluxes, with errors small compared to signal size, and in particular compared to the intrinsic inversion errors as revealed by the deviations of the benchmark from the "known truth" in test A. However, these results are specific to DoI, data, set-up, transport model, and target quantities. How general is this behaviour?

- In our examples, it is certainly advantageous that the western (inflow) boundary of the DoI lies in the ocean, such that no correlations of the land fluxes are disrupted there. In general, therefore, if an inversion targets at land(ocean) fluxes, the DoI boundary should as far as possible cut in the ocean(land).

- Nesting makes naturally most sense enclosing an area with relatively high density of observation sites, able to sample the mixing ratio gradients within the DoI. The DoI boundary should not be too close to any site, such that the trans-contribution of the mixing ratio smoothes out more.

- Edge effects on the a-priori correlations are less severe with shorter correlation lengths. When the tests were done using an inversion set-up with longer spatial correlations, the performance of the scheme indeed deteriorated. Choosing shorter a-priori correlations is thus advisable.

- Though the presented tests use related transport models in steps 1 and 2, the scheme itself does not make any assumptions on the nature and similarity of $\mathbf{A}_{\text {coarse }}^{\text {glob }}$ and $\mathbf{A}_{\text {fine }}^{\text {reg }}$. The coupling only happens on the level of mixing ratio vectors $\boldsymbol{c}$, no matter how they are represented in the respective models. Therefore, we expect the scheme to work with other pairs of models as well, including Eulerian and Lagrangian ones.

In fact, the presented choice of test models represents a rather harsh ordeal of the scheme, because the coarse model performs actually quite poorly in representing the fine-structure of the continental mixing ratio field (see the mixing ratio residuals in Fig. 7, blue line). In a real application, the model for step 1 would of course be chosen as fine as computationally feasible (e.g., the standard $\approx 4^{\circ} \times 5^{\circ}$ resolution variant of TM3).

Numerically testing the scheme for a given pair of transport models is, of course, difficult as soon as the benchmark becomes computationally infeasible. As a potential work-around, one could do the inversion in three steps (global - larger DoI - smaller DoI), and try to perform the middle step both on coarse and fine resolution, thereby creating a partial benchmark for the third step.

- The target quantities of the presented tests (regionally integrated and possibly filtered fluxes) are common output quantities of inversion studies, but other features of the flux may be of interest as well. Analogous tests could be done for any target quantity. However, we expect that the ratio between the additional errors from the two-step scheme and the intrinsic inversion errors would stay at a similar order of magnitude, as is the case among the individual integration regions shown here. (Note that errors generally rise for smaller integration regions.)

\subsection{Computational gain}

The aim of any nesting scheme is to provide an approximation to a hypothetical high-resolution global inversion, where CPU demands are sufficiently reduced to make the calculation feasible (while the additional errors remain sufficiently small). The computational gain arises here

- because the spin-up period is mainly taken care of by the coarse-resolution step 1 , such that the PoI can be shorter;

- because less iterations are needed for convergence of the iterative solution (in test $\mathrm{A}$, less than 20 rather than 30 to 40 ), due to the smaller number of constraints to be simultaneously satisfied;

- and, most importantly, because the regional model only has to be run within the DoI.

\subsection{Calculating 3-D mixing ratio fields}

A forward model run based on the flux results of an (ordinary) inversion does not only yield mixing ratio values $\boldsymbol{c}_{\text {mod }_{1}}$ at the locations and times of the data, but a full 3-D mixing ratio field $c_{\bmod _{1}}^{\text {glob }}(\boldsymbol{x}, t)$ (that, in analogy to weather forecasting, could be called "analyzed" or "assimilated" mixing ratios). In the case of the two-step scheme with an Eulerian regional model, the corresponding 3-D mixing ratio field within the DoI is the sum of the trans-contribution calculated from step 1 and the (cis-) contribution from step 2,

$c_{\bmod _{2}}^{\mathrm{reg}}(\boldsymbol{x}, t)=c_{\bmod _{1}, \text { trans }}^{\mathrm{reg}}(\boldsymbol{x}, t)+c_{\bmod _{2}, \mathrm{cis}}^{\mathrm{reg}}(\boldsymbol{x}, t)$

where $c_{\text {mod }_{1} \text {,trans }}^{\text {reg }}(\boldsymbol{x}, t)$ can be obtained as a difference between full global fields and their cis-part.

\section{Conclusions}

This paper introduces a two-step scheme for high-resolution flux estimation, by spatio-temporal nesting of a domain of 

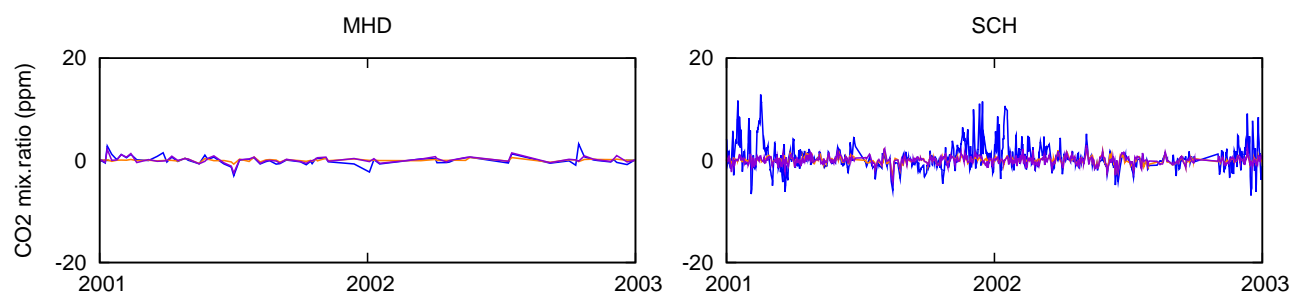

Fig. 7. Comparison of the residuals of the step-2 inversion $\left(\boldsymbol{c}_{\text {meas }}-\boldsymbol{c}_{\bmod _{2}}\right.$, violet) with those of the benchmark inversion $\left(\boldsymbol{c}_{\mathrm{meas}}-\boldsymbol{c}_{\mathrm{mod}}\right.$, orange) and of the step-1 inversion ( $\boldsymbol{c}_{\text {meas }}-\boldsymbol{c}_{\text {mod }_{1}}$, blue).

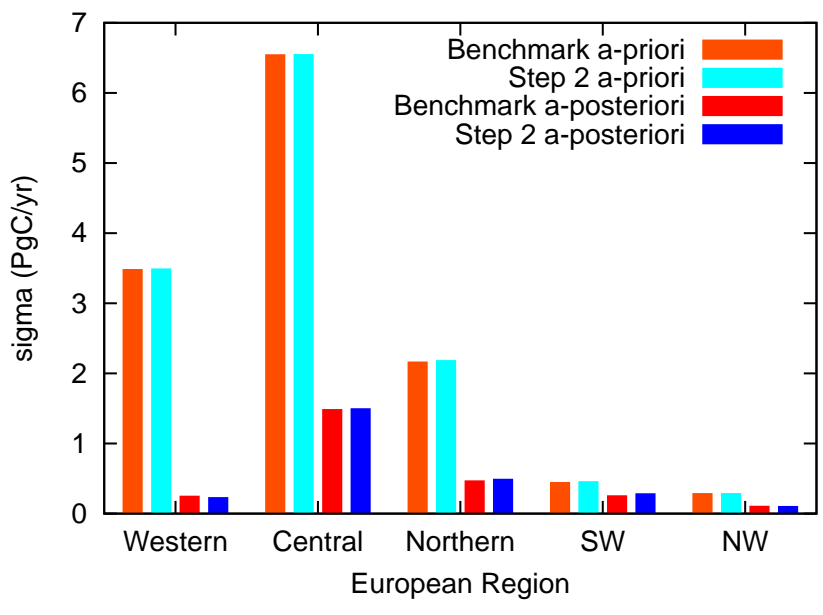

Fig. 8. A-priori and a-posteriori uncertainty intervals for regionally integrated and temporally filtered fluxes (as in Fig. 3), as calculated by the benchmark inversion and the regional approximation.

interest (DoI) and a period of interest (PoI) into a global and longer inversion. It does not require any zooming or nesting capabilities in modelling atmospheric transport. Only relatively minor work is needed to implement the scheme into an existing (single-model) inversion system:

- The global transport model needs the option to only simulate the cis-contribution within the DoI, e.g., by setting the mixing ratio field outside the DoI to zero after each model time step;

- suitable logic in the inversion system to replace the data by a difference of modelled mixing ratios $\left(\Delta \boldsymbol{c}_{\text {remain }}\right)$;

- options to suitably manipulate the a-priori settings of the inversion to accommodate the cut-out spatial and temporal domain (see Appendix B).

Once done, essentially any pair of transport models can technically be nested. Potentially, also a further refinement by adding more steps on even higher resolution may be appropriate.
According to our test examples, the two-step nesting scheme approximates, within the domain of interest, the results of a hypothetical global high-resolution inversion with for practical purposes fully sufficient accuracy. The functioning of the scheme can be understood mathematically in terms of a split of the mixing ratio field within the regional domain into contributions reflecting the local fluxes and smooth contributions advected from the boundary.

\section{Appendix A}

The (global) mixing ratio field $c^{\text {glob }}(\boldsymbol{x}, t)$ of a conservative atmospheric tracer, given its sources and sinks ("fluxes") $F(x, t)$ and the atmospheric transport, is a solution of the three-dimensional, time-dependent continuity equation

$\mathcal{T}\left[c^{\text {glob }}(\boldsymbol{x}, t)\right]=F(\boldsymbol{x}, t)$

with initial conditions

$c^{\text {glob }}(\boldsymbol{x}, t=0)=c_{\text {ini }}(\boldsymbol{x})$

The transport operator $\mathcal{T}$ is given by

$\mathcal{T}\left[{ }_{-}\right]=\frac{\partial}{\partial t} \varrho(\boldsymbol{x}, t)[-]+\nabla \cdot \boldsymbol{v}(\boldsymbol{x}, t) \varrho(\boldsymbol{x}, t)[-]$

where $\varrho$ denotes the air density (moles per unit volume) and $v$ the 3-D wind vector ${ }^{6}$. In the present context, the fluxes will mainly be surface sources/sinks,

$F(\boldsymbol{x}, t)=\delta(z) f(\boldsymbol{x}, t)$

with Dirac's delta $\delta$, the distance $z$ from the surface, and the 2-D fluxes $f(\boldsymbol{x}, t)$.

If the consideration is restricted to the domain of interest (DoI) and the period of interest (PoI), the (regional) mixing ratio field $c^{\text {reg }}(\boldsymbol{x}, t)$ within the DoI/PoI is a solution of the same continuity equation Eq. (A1), with the regional fluxes only, but additional sources/sinks along the boundaries,

$\mathcal{T}\left[c^{\mathrm{reg}}\right]=F^{\mathrm{reg}}+V_{\text {in } \varrho} c^{\text {glob }}+V_{\text {out }} \varrho c^{\mathrm{reg}}$

\footnotetext{
${ }^{6}$ The discrete representation of this operator in gridded numerical transport models would additionally involve diffusional terms to parametrize sub-grid processes. As such terms are linear as well, however, they do not affect the discussion.
} 
where the second and third terms on the r.h.s. represent inflow from the global domain and outflow out of the DoI, respectively, ensuring that $c^{\text {reg }}(\boldsymbol{x}, t)$ is identical to $c^{\text {glob }}(\boldsymbol{x}, t)$ within the DoI. Here we have introduced the transfer rate $V(\boldsymbol{x}, t)$ perpendicular to the boundaries,

$V:=\delta(n) \boldsymbol{n} \cdot \boldsymbol{v}$

(with $n$ the distance from the closest boundary and $\boldsymbol{n}$ the normal vector pointing into the DoI), split into its positive and negative parts,

$V_{\text {in }}:=\left\{\begin{array}{c}V, V>0 \\ 0, V \leq 0\end{array} \quad V_{\text {out }}:=\left\{\begin{array}{c}0, V>0 \\ V, V \leq 0\end{array}\right.\right.$

The trans- and cis-contributions can now be represented as the solutions to

$\mathcal{T}\left[c_{\text {trans }}^{\text {reg }}\right]=\quad V_{\text {in }} \varrho c^{\text {glob }}+V_{\text {out }} \varrho c_{\text {trans }}^{\text {reg }}$

and

$\mathcal{T}\left[c_{\text {cis }}^{\text {reg }}\right]=F^{\text {reg }} \quad+V_{\text {out }} \varrho c_{\text {cis }}^{\text {reg }}$

respectively ${ }^{7}$.

As discussed, the trans-contribution does not directly depend on the regional fluxes $F^{\text {reg }}(\boldsymbol{x}, t)$, except for their impact on the global background contained in $c^{\text {glob }}(\boldsymbol{x}, t)$.

A regional transport model without any tracer inflow simulates $c_{\text {cis }}^{\text {reg }}(\boldsymbol{x}, t)$. The same behaviour can be emulated in a global model by setting the mixing ratio field to zero after each step: inflow will be zero (missing term $V_{\text {in } \varrho c^{\text {glob }}}$ in Eq. A9), and any outflowing mixing ratio is cancelled (negative-velocity term $V_{\text {out }} \varrho c_{\text {cis }}^{\text {reg }}$ ). This is what has been done to obtain the regional coarse-grid model $\mathbf{A}_{\text {coarse }}^{\text {reg }}(\mathrm{Ta}-$ ble 1).

\section{Appendix B}

In the implementation of the step-2 inversion, some secondary changes with respect to the usual inversion procedure are necessary:

- As the fluxes are only defined within the DoI, suitable modifications to the a-priori information are required along the boundary.

In the flux model formulation used here (Rödenbeck, 2005), all the elementary spatio-temporal flux patterns (the columns of matrix F, Eq. 3) are cut at the boundary,

\footnotetext{
an analogous split,

$c^{\mathrm{reg}}(\boldsymbol{x}, t=\partial \mathrm{PoI})=c^{\text {glob }}(\boldsymbol{x}, t=\partial \mathrm{PoI})$

$c_{\text {trans }}^{\text {reg }}(\boldsymbol{x}, t=\partial \mathrm{PoI})=c^{\text {glob }}(\boldsymbol{x}, t=\partial \mathrm{PoI})$

$c_{\text {cis }}^{\text {reg }}(\boldsymbol{x}, t=\partial \mathrm{PoI})=0$
}

${ }^{7}$ The initial conditions at the beginning $\partial \mathrm{PoI}$ of the PoI involve such that the spatial correlations from inside to outside the DoI are disrupted. However, the point-wise normalization of $\mathbf{F} \boldsymbol{p}$ restores the pattern of a-priori sigma intervals of the individual pixels. As our implementation also involves a normalization according to the a-priori sigmas of specific linear flux functionals (that extend beyond the DoI and short PoI's), corrective scaling factors had to be applied to ensure that the pixel-wise apriori sigmas are equal to that in the global flux model.

- The inversion runs only over the PoI (implying minor changes to the temporal correlation structure near the start/end of the PoI).

- The initial mixing ratio (at the start of the PoI) is zero, and any degrees of freedom related to adjustments of the initial mixing ratio are removed.

Note: In the step-2 inversion, both data and fluxes are only defined within the DoI/PoI. In the numerical implementation, however, it does not harm to keep the data outside the DoI because this would just add a constant offset to the cost function $J_{c}$ irrelevant to the minimization. Likewise, if convenient, the flux fields may be implemented globally because any fluxes outside the DoI would not interfere with the step- 2 estimation.

Acknowledgements. We would like to thank T. Aalto, F. Artuso, G. Carboni, T. J. Conway, L. Haszpra, A. Jordan, C. Labuschagne, R. Langenfelds, I. Levin, T. Machida, F. Meinhardt, T. Nakazawa, E. Popa, M. Ramonet, L. Rivier, R. Santaguida, A. di Sarra, M. Schmidt, A. Vermeulen, D. Worthy, M. Zimnoch, and all other colleagues and their institutions involved in the collection and measurement of the atmospheric data. We would like to thank S. Mikaloff-Fletcher for kindly providing the oceanic fluxes used to create the pseudo-data. Stimulating discussions with P. Peylin, S. Houweling, and P. Rayner are gratefully acknowledged.

The service charges for this open access publication have been covered by the Max Planck Society.

Edited by: A. Stohl

\section{References}

Ahmadov, R., Gerbig, C., Kretschmer, R., et al: Mesoscale covariance of transport and $\mathrm{CO}_{2}$ fluxes: Evidence from observations and simulations using the WRF-VPRM coupled atmospherebiosphere model, J. Geophys. Res.-Atmos., 112, D22107, doi:10.1029/2007JD008 552, 2007.

Baker, D. F., Law, R. M., Gurney, K. R., Rayner, P., Peylin, P., Denning, A. S., Bousquet, P., Bruhwiler, L., Chen, Y.-H., Ciais, P., Fung, I. Y., Heimann, M., John, J., Maki, T., Maksyutov, S., Masarie, K., Prather, M., Pak, B., Taguchi, S., and Zhu, Z.: TransCom 3 inversion intercomparison: Impact of transport model errors on the interannual variability of regional $\mathrm{CO}_{2}$ fluxes, 1988-2003, Global Biogeochem. Cy., 20, GB1002, doi:10.1029/2004GB002439, 2006. 
Bousquet, P., Peylin, P., Ciais, P., Le Quéré, C., Friedlingstein, P., and Tans, P. P.: Regional changes in carbon dioxide fluxes of land and oceans since 1980, Science, 290, 1342-1346, 2000.

BP: Statistical Review of World Energy June 2006, online available at: http://www.bp.com/statisticalreview.

Enting, I., Trudinger, C. M., and Francey, R. J.: A synthesis inversion of the concentration and $\delta^{13} \mathrm{C}$ of atmospheric $\mathrm{CO}_{2}$, Tellus, 47B, 35-52, 1995.

Geels, C., Gloor, M., Ciais, P., Bousquet, P., Peylin, P., Vermeulen, A. T., Dargaville, R., Aalto, T., Brandt, J., Christensen, J. H., Frohn, L. M., Haszpra, L., Karstens, U., Rödenbeck, C., Ramonet, M., Carboni, G., and Santaguida, R.: Comparing atmospheric transport models for future regional inversions over Europe Part 1: mapping the atmospheric $\mathrm{CO}_{2}$ signals, Atmos. Chem. Phys., 7, 3461-3479, 2007,

http://www.atmos-chem-phys.net/7/3461/2007/.

Gerbig, C., Lin, J., Wofsy, S., Daube, B. C., Andrews, A. E., Stephens, B. B., Bakwin, P. S., and Grainger, C. A.: Toward constraining regional-scale fluxes of $\mathrm{CO}_{2}$ with atmospheric observations over a continent: 1 . Observed spatial variability from airborne platforms, J. Geophys. Res.-Atmos., 108, 4756, doi: 10.1029/2002JD003018, 2003a.

Gerbig, C., Lin, J., Wofsy, S., Daube, B. C., Andrews, A. E., Stephens, B. B., Bakwin, P. S., and Grainger, C. A.: Toward constraining regional-scale fluxes of $\mathrm{CO}_{2}$ with atmospheric observations over a continent: 2. Analysis of COBRA data using a receptor-oriented framework, J. Geophys. Res.-Atmos., 108, 4757, doi:10.1029/2003JD003770, 2003b.

Gloor, M., Gruber, N., Sarmiento, J., Sabine, C. L., Feely, R. A., and Rödenbeck, C.: A first estimate of present and preindustrial air-sea $\mathrm{CO}_{2}$ flux patterns based on ocean interior carbon measurements and models, Geophys. Res. Lett., 30, 1010, doi:10.1029/2002GL015 594, 2003.

Heimann, M. and Körner, S.: The global atmospheric tracer model TM3, Tech. Rep. 5, MPI BGC, Jena (Germany), online available at: http://www.bgc-jena.mpg.de/mpg/websiteBiogeochemie/ Publikationen/Technical_Reports/tech_report5.pdf, 2003.

Jacobson, A. R., Fletcher, S. E. M., Gruber, N., Sarmiento, J. L., and Gloor, M.: A joint atmosphere-ocean inversion for surface fluxes of carbon dioxide, I: Methods and global-scale fluxes, Global Biogeochem. Cy., 21, GB1019, doi:10.1029/2005GB002 556, 2007.

Kalnay, E., Kanamitsu, M., Kistler, R., Collins, W., Deaven, D., Gandin, L., Iredell, M., Saha, S., White, G., Woollen, J., Zhu, Y., Chelliah, M., Ebisuzaki, W., Higgins, W., Janowiak, J., Mo, K. C., Ropelewski, C., Wang, J., Leetmaa, A., Reynolds, R., Jenne, R., and Joseph, D.: The NCEP/NCAR 40-year reanalysis project, B. Am. Meteor. Soc., 77, 437-471, 1996.

Lauvaux, T., Uliasz, M., Sarrat, C., Chevallier, F., Bousquet, P., Lac, C., Davis, K. J., Ciais, P., Denning, A. S., and Rayner, P. J.: Mesoscale inversion: first results from the CERES campaign with synthetic data, Atm. Chem. Phys., 8, 3459-3471, 2008.

Lin, J., Gerbig, C., Wofsy, S., and et al: A near-field tool for simulating the upstream influence of atmospheric observations: The Stochastic Time-Inverted Lagrangian Transport (STILT) model, J. Geophys. Res.-Atmos., 108, 4493, doi:10. 1029/2002JD003161, 2003.

Mikaloff Fletcher, S. E., Gruber, N., Jacobson, A. R., Doney, S. C., Dutkiewicz, S., Gerber, M., Follows, M., Joos, F., Lindsay, K.,
Menemenlis, D., Mouchet, A., Müller, S. A., and Sarmiento, J. L.: Inverse estimates of anthropogenic $\mathrm{CO}_{2}$ uptake, transport, and storage by the ocean, Global Biogeochem. Cy., 20, GB2002, doi:10.1029/2005GB002530, 2006.

Mikaloff Fletcher, S. E., Gruber, N., Jacobson, A. R., Doney, S. C., Dutkiewicz, S., Gerber, M., Gloor, M., Follows, M., Joos, F., Lindsay, K., Menemenlis, D., Mouchet, A., Müller, S. A., and Sarmiento, J. L.: Inverse estimates of the oceanic sources and sinks of natural $\mathrm{CO}_{2}$ and the implied oceanic transport, Global Biogeochem. Cy., 21, GB1010, doi:10.1029/2006GB002 751, 2007.

Olivier, J. G. J. and Berdowski, J. J. M.: Global emissions sources and sinks, in: The Climate System, edited by: Berdowski, J., Guicherit, R. and Heij, B. J., A. A. Balkema Publishers/Swets \& Zeitlinger Publishers, Lisse, The Netherlands, ISBN: 90-5809255-0, 33-78, 2001.

Pérez-Landa, G., Ciais, P., Gangoiti, G., Palau, J. L., Carrara, A., Gioli, B., Miglietta, F., Schumacher, M., Millán, M. M., and Sanz, M. J.: Mesoscale circulations over complex terrain in the Valencia coastal region, Spain - Part 2: Modeling $\mathrm{CO}_{2}$ transport using idealized surface fluxes, Atmos. Chem. Phys., 7, 18511868, 2007, http://www.atmos-chem-phys.net/7/1851/2007/.

Peters, W., Jacobson, A. R., Sweeney, C., Andrews, A. E., Conway, T. J., Masarie, K., Miller, J. B., Bruhwiler, L. M. P., Pétron, G., Hirsch, A. I., Worthy, D. E. J., van der Werf, G. R., Randerson, J. T., Wennberg, P. O., Krol, M. C., and Tans, P. P.: An atmospheric perspective on North American carbon dioxide exchange: CarbonTracker, PNAS, 104, 18925-18930, 2007.

Peylin, P., Rayner, P. J., Bousquet, P., Carouge, C., Hourdin, F., Heinrich, P., Ciais, P., and AEROCARB contributors: Daily $\mathrm{CO}_{2}$ flux estimates over Europe from continuous atmospheric measurements: 1, inverse methodology, Atm. Chem. Phys., 5, 31733186, 2005.

Rödenbeck, C.: Estimating $\mathrm{CO}_{2}$ sources and sinks from atmospheric mixing ratio measurements using a global inversion of atmospheric transport, Tech. Rep. 6, MPI BGC, Jena (Germany), online available at: http://www.bgc-jena.mpg.de/mpg/websiteBiogeochemie/ Publikationen/Technical_Reports/tech_report6.pdf, 2005.

Rödenbeck, C., Houweling, S., Gloor, M., and Heimann, M.: $\mathrm{CO}_{2}$ flux history 1982-2001 inferred from atmospheric data using a global inversion of atmospheric transport, Atm. Chem. Phys., 3, 1919-1964, 2003.

Sitch, S., Prentice, I., Smith, B., Cramer, W., Kaplan, J., Lucht, W., Sykes, M., Thonicke, K., and Venevsky, S.: LPJ - a coupled model of vegetation dynamics and the terrestrial carbon cycle, in: The role of vegetation dynamics in the control of atmospheric $\mathrm{CO}_{2}$ content, edited by: Sitch, S., PhD Thesis, Lund University, Lund, Sweden, 2000.

Trusilova, K. and Churkina, G.: The terrestrial ecosystem model BIOME-BGC v1, Tech. Rep. 14, MPI BGC, Jena (Germany), online availble at: http://www.bgc-jena.mpg.de/ mpg/websiteBiogeochemie/Publikationen/Technical_Reports/ tech_report14.pdf, 2008.

van der Molen, M. and Dolman, H.: Regional carbon fluxes and the effect of topography on the variability of atmospheric $\mathrm{CO}_{2}$, J. Geophys. Res.-Atmos., 112, D01104, doi:10.1029/2006JD007 649, 2007. 